\title{
AN EXPLICIT FORMULA FOR BERNOULLI POLYNOMIALS IN TERMS OF $r$-STIRLING NUMBERS OF THE SECOND KIND
}

\author{
BAI-NI GUO, ISTVÁN MEZŐ AND FENG QI
}

\begin{abstract}
In this paper, the authors establish an explicit formula for computing Bernoulli polynomials at nonnegative integer points in terms of $r$-Stirling numbers of the second kind.
\end{abstract}

1. Introduction. It is well known that the Bernoulli numbers $B_{k}$ for $k \geq 0$ can be generated by

$$
\frac{t}{e^{t}-1}=\sum_{k=0}^{\infty} B_{k} \frac{t^{k}}{k !}=1-\frac{t}{2}+\sum_{k=1}^{\infty} B_{2 k} \frac{t^{2 k}}{(2 k) !}, \quad|t|<2 \pi,
$$

and that the Bernoulli polynomials $B_{n}(x)$ for $n \geq 0$ and $x \in \mathbb{R}$ can be generated by

$$
\frac{t e^{x t}}{e^{t}-1}=\sum_{n=0}^{\infty} B_{n}(x) \frac{t^{n}}{n !}, \quad|t|<2 \pi
$$

In combinatorics, Stirling numbers of the second kind $S(n, k)$ are equal to the number of partitions of the set $\{1,2, \ldots, n\}$ into $k$ nonempty disjoint sets. Stirling numbers of the second kind $S(n, k)$ for $n \geq k \geq 0$ can be computed by

$$
S(n, k)=\frac{1}{k !} \sum_{\ell=0}^{k}(-1)^{k-\ell}\left(\begin{array}{l}
k \\
\ell
\end{array}\right) \ell^{n} .
$$

In [1], Stirling numbers $S(n, k)$ were combinatorially generalized as $r$-Stirling numbers of the second kind, denoted by $S_{r}(n, k)$ here, for $r \in \mathbb{N}$, which can alternatively be defined as the number of partitions

2010 AMS Mathematics subject classification. Primary 11B73, Secondary $05 \mathrm{~A} 18$.

Keywords and phrases. Explicit formula, Bernoulli number, Bernoulli polynomial, Stirling number of the second kind, $r$-Stirling number of the second kind.

Received by the editors on March 1, 2015. 
of the set $\{1,2, \ldots, n\}$ into $k$ nonempty disjoint subsets such that the numbers $1,2, \ldots, r$ are in distinct subsets.

Note that

$$
\begin{array}{ll}
S(0,0)=1, & S_{0}(n, k)=S(n, k), \\
S(n, 0)=0, & S_{1}(n, k)=S(n, k)
\end{array}
$$

for all $n \geq k \geq 0$.

In [4, page 536] and [5, page 560], the simple formula

$$
B_{n}=\sum_{k=0}^{n}(-1)^{k} \frac{k !}{k+1} S(n, k), \quad n \in \mathbb{N} \cup\{0\}
$$

for computing the Bernoulli numbers $B_{n}$ in terms of Stirling numbers of the second kind $S(n, k)$ was incidentally obtained. Recently, four alternative proofs for formula (1.2) were supplied in [6, 7, 16]. For more information on calculation of the Bernoulli numbers $B_{n}$, please refer to $[\mathbf{8}, \mathbf{9}, \mathbf{1 0}, \mathbf{1 1}, \mathbf{1 3}, \mathbf{1 5}, \mathbf{1 7}]$, especially to $[\mathbf{3}]$, and the many references therein.

The aim of this paper is to generalize formula (1.2). Our main result can be formulated as the following theorem.

Theorem 1.1. For all integers $n, r \geq 0$, the Bernoulli polynomials $B_{n}(r)$ can be computed in terms of $r$-Stirling numbers of the second kind $S_{r}(n+r, k+r)$ by

$$
B_{n}(r)=\sum_{k=0}^{n}(-1)^{k} \frac{k !}{k+1} S_{r}(n+r, k+r) .
$$

In the final section of this paper, several remarks are listed.

2. Proof of Theorem 1.1. We are now in a position to verify our main result.

For $n, r \geq 0$, let

$$
F_{n, r}(x)=\sum_{k=0}^{n} k ! S_{r}(n+r, k+r) x^{k} .
$$


By [1, page 250, Theorem 16], we have

$$
\begin{aligned}
\sum_{n=0}^{\infty} S_{r}(n+r, k+r) \frac{t^{n}}{n !} & =\sum_{n=k}^{\infty} S_{r}(n+r, k+r) \frac{t^{n}}{n !} \\
& =\frac{1}{k !} e^{r t}\left(e^{t}-1\right)^{k},
\end{aligned}
$$

where $S_{r}(n, m)=0$ for $m>n$, see [1, page 243, equation (10)]. Accordingly, we obtain

$$
\begin{aligned}
\sum_{n=0}^{\infty} F_{n, r}(x) \frac{t^{n}}{n !} & =\sum_{n=0}^{\infty} \sum_{k=0}^{n} k ! x^{k} S_{r}(n+r, k+r) \frac{t^{n}}{n !} \\
& =\sum_{k=0}^{\infty} k ! x^{k} \sum_{n=k}^{\infty} S_{r}(n+r, k+r) \frac{t^{n}}{n !} \\
& =e^{r t} \sum_{k=0}^{\infty} x^{k}\left(e^{t}-1\right)^{k}=\frac{e^{r t}}{1-x\left(e^{t}-1\right)} .
\end{aligned}
$$

For $s \in \mathbb{R}$, integrating with respect to $x \in[0, s]$ on both sides of the above equation yields

$$
\sum_{n=0}^{\infty}\left[\int_{0}^{s} F_{n, r}(x) \mathrm{d} x\right] \frac{t^{n}}{n !}=-e^{r t} \frac{\ln \left(1+s-s e^{t}\right)}{e^{t}-1} .
$$

On the other hand,

$$
\int_{0}^{s} F_{n, r}(x) \mathrm{d} x=\sum_{k=0}^{n} \frac{k !}{k+1} S_{r}(n+r, k+r) s^{k+1} .
$$

Substituting this into equation (2.1) gives

$$
\sum_{n=0}^{\infty} \sum_{k=0}^{n} \frac{k !}{k+1} S_{r}(n+r, k+r) s^{k+1} \frac{t^{n}}{n !}=-e^{r t} \frac{\ln \left(1+s-s e^{t}\right)}{e^{t}-1} .
$$

Taking $s=-1$ in the above equation and using the generating function (1.1) results in

$$
\sum_{n=0}^{\infty}\left[\sum_{k=0}^{n}(-1)^{k+1} \frac{k !}{k+1} S_{r}(n+r, k+r)\right] \frac{t^{n}}{n !}=-\frac{t e^{r t}}{e^{t}-1}=\sum_{n=0}^{\infty}\left[-B_{n}(r)\right] \frac{t^{n}}{n !},
$$

which implies formula (1.3). The proof of Theorem 1.1 is complete. 
3. Remarks. Finally, we would like to give several remarks on Theorem 1.1 and its proof.

Remark 3.1. Since $B_{n}(0)=B_{n}$ and $S_{0}(n, k)=S(n, k)$, when $r=0$, formula (1.3) becomes (1.2). Therefore, our Theorem 1.1 generalizes formula (1.2).

Remark 3.2. It is easy to see that

$$
F_{n, 0}(1)=\sum_{k=0}^{n} k ! S(n, k),
$$

which are the classical ordered Bell numbers. For more information, please refer to $[\mathbf{2}, \mathbf{1 4}]$ and the closely related references therein.

Remark 3.3. In [12], the second author defined a variant of the polynomials $F_{n, r}(x)$. Hence, a simple combinatorial study and interpretation of the polynomials $F_{n, r}(x)$ is available therein.

\section{REFERENCES}

1. A.Z. Broder, The r-Stirling numbers, Discrete Math. 49 (1984), 241-259.

2. M.B. Can and M. Joyce, Ordered Bell numbers, Hermite polynomials, skew Young tableaux, and Borel orbits, J. Combin. Theory 119 (2012), 1798-1810.

3. H.W. Gould, Explicit formulas for Bernoulli numbers, Amer. Math. Month. 79 (1972), 44-51.

4. R.L. Graham, D.E. Knuth and O. Patashnik, Concrete mathematics-A foundation for computer science, Addison-Wesley Publishing Company, Advanced Book Program, Reading, MA, 1989.

5. Concrete mathematics-A foundation for computer science, 2nd edition, Addison-Wesley Publishing Company, Reading, MA, 1994.

6. B.-N. Guo and F. Qi, A new explicit formula for the Bernoulli and Genocchi numbers in terms of the Stirling numbers, Glob. J. Math. Anal. 3 (2015), 33-36.

7. Alternative proofs of a formula for Bernoulli numbers in terms of Stirling numbers, Analysis 34 (2014), 187-193

8. , An explicit formula for Bernoulli numbers in terms of Stirling numbers of the second kind, J. Anal. Num. Th. 3 (2015), 27-30.

9. __ Explicit formulae for computing Euler polynomials in terms of Stirling numbers of the second kind, J. Comp. Appl. Math. 272 (2014), 251-257.

10. , Some identities and an explicit formula for Bernoulli and Stirling numbers, J. Comp. Appl. Math. 255 (2014), 568-579. 
11. S.-L. Guo and F. Qi, Recursion formulae for $\sum_{m=1}^{n} m^{k}$, Z. Anal. Anwend. 18 (1999), 1123-1130.

12. I. Mező, Combinatorial interpretation of some combinatorial numbers, Ph.D. dissertation, University of Debrecen, Hungary, 2010 (in Hungarian).

13. F. Qi, A double inequality for ratios of the Bernoulli numbers, ResearchGate Dataset, available online at http://dx.doi.org/10.13140/RG.2.1.3461.2641.

14. , An explicit formula for the Bell numbers in terms of the Lah and Stirling numbers, Mediterranean J. Math. 13 (2016), 2795-2800.

15. Derivatives of tangent function and tangent numbers, Appl. Math. Comp. 268 (2015), 844-858.

16. F. Qi and B.-N. Guo, Alternative proofs of a formula for Bernoulli numbers in terms of Stirling numbers, Analysis 34 (2014), 311-317.

17. A.-M. Xu and Z.-D. Cen, Some identities involving exponential functions and Stirling numbers and applications, J. Comp. Appl. Math. 260 (2014), 201-207.

School of Mathematics and Informatics, Henan Polytechnic University, Jiaozuo City, Henan Province, 454010, China

Email address: bai.ni.guo@gmail.com, bai.ni.guo@hotmail.com

Department of Mathematics, Nanjing University of Information Science And Technology, Nanjing City, 210044, China

Email address: istvanmezo81@gmail.com

Department of Mathematics, College of Science, Tianjin Polytechnic University, Tianjin City, 300387, China

Email address: qifeng618@gmail.com, qifeng618@hotmail.com 